\title{
A SPLITTING THEOREM ON TORIC MANIFOLDS
}

\author{
HONGNIAN HUANG
}

\begin{abstract}
Using the Calabi flow, we prove that any extremal Kähler metric $\omega_{E}$ on a product toric variety $X_{1} \times X_{2}$ is a product extremal Kähler metric.
\end{abstract}

\section{Introduction}

In [3], the authors considered the following problem:

Problem 1.1. Let $X_{i}, i=1,2$ be two Kähler manifolds with Kähler classes $\left[\omega_{i}\right]$. Suppose $\omega_{E}$ is an extremal Kähler metric in the Kähler class $\left[\omega_{1}+\omega_{2}\right]$. Can we conclude that $\omega_{E}$ is a product metric, i.e., $\omega_{E}=\omega_{E, 1}+\omega_{E, 2}$ where $\omega_{E, i}$ is an extremal Kähler metric in $\left[\omega_{i}\right]$.

In this short paper, we solve the problem in the case of toric manifolds.

Theorem 1.2. If $X_{i}$ are toric manifolds, then $\omega_{E}$ is a product metric.

\section{Motivations and setup}

Let $X$ be a $n$-dimensional Kähler manifold with Kähler class [ $\omega]$. The set of relative Kähler potentials is

$$
\mathcal{H}=\left\{\varphi \in C^{\infty}(X) \mid \omega_{\varphi}=\omega+i \partial \bar{\partial} \varphi>0\right\} .
$$

An extremal Kähler metric $\omega_{\varphi}$ in the sense of Calabi is defined by the condition that its scalar curvature $R_{\varphi}$ is a potential of a Killing vector field of $\left(X, \omega_{\varphi}\right)$. In [5], Calabi proved that any extremal metric is invariant under a maximal compact subgroup of the reduced automoprhism group of $X$. As any such groups are conjugated, one can fix the isometry group of an extremal Kähler metric. In particular, if we further assume that $X$ is a toric manifold, then without loss of generality we can assume that if an extremal metric $\omega_{E}$ exists then it is invariant under the (real) torus $\mathbb{T}^{n}$. We thus focus on the space of $\mathbb{T}^{n}$-invariant relative Kähler potentials:

$$
\mathcal{H}_{\mathbb{T}^{n}}=\left\{\varphi \in C^{\infty}(X) \mid \varphi \text { is invariant under } \mathbb{T}^{n}, \omega_{\varphi}=\omega+i \partial \bar{\partial} \varphi>0\right\},
$$

where $\omega$ is a $\mathbb{T}^{n}$-invariant Kähler metric on $X$. By the equivariant Moser lemma, the space of $\mathbb{T}^{n}$-invariant Kähler metrics $\omega_{\varphi}$ given by elements in $\mathcal{H}_{\mathbb{T}^{n}}$ can be alternatively realized as the space $\mathcal{J}_{\mathbb{T}^{n}}^{\omega}$ of $\mathbb{T}^{n}$-invariant $\omega$-compatible complex structures on the toric symplectic manifold $(X, \omega)$, see Abreu [2], Guillemin [19,20] and Donaldson [9]. The latter is identified, via the momentum map, to the corresponding Delzant polytope $P \subset \mathbb{R}^{n}$ (see Delzant [16]). Recall that $P$ is a compact convex polytope satisfying

Received by the editors November 2, 2012. 
the following conditions:

- For any facet $P_{i}$ of $P$, there exists an inward normal vector $\vec{v}_{i}$ corresponding to $P_{i}$.

- For any vertex $v$ of $P$, there are exactly $n$ facets $P_{1}, \ldots, P_{n}$ meeting at $v$ and the inward normal vectors $\vec{v}_{1}, \ldots, \vec{v}_{n}$ form a basis of $\mathbb{Z}^{n}$.

Suppose that $P$ has $d$-facets. For every facet $P_{i}$, we choose $c_{i}$ such that $l_{i}(x)=$ $\left\langle x, \vec{v}_{i}\right\rangle+c_{i}$ vanishes on $P_{i}$.

Definition 2.1. A smooth strictly convex function $u$ on the interior of $P$ is called a symplectic potential if

- $u$ extends as a continuous function over $\partial P$ and its restriction to the interior of each face of $P$ is smooth and strictly convex;

$$
u(x)=\sum_{i=1}^{d} \frac{1}{2} l_{i}(x) \ln l_{i}(x)+f(x),
$$

where $f(x)$ is a smooth function up to the boundary of $P$.

The main point of this definition is that, for appropriate choice of angular coordinates $\left(t_{1}, \ldots, t_{n}\right)$, the almost complex structure

$$
J_{u}=\left(\begin{array}{ccc}
0 & \vdots & -\left(D^{2} u\right)^{-1} \\
\cdots & \cdots & \cdots \\
\left(D^{2} u\right) & \vdots & 0
\end{array}\right)
$$

is an element of $\mathcal{J}_{\mathbb{T}^{n}}^{\omega}$ and the elements of $\mathcal{H}_{\mathbb{T}^{n}}$ are in one to one correspondence with symplectic potentials $u$ as above, see $[2,9,19,20]$.

Furthermore, Abreu [1] wrote down the expression of the scalar curvature $R_{u}$ of the Kähler metric $g_{u}(\cdot, \cdot)=\omega\left(\cdot, J_{u} \cdot\right)$. It follows that a symplectic potential $u_{E}$ corresponds to an extremal Kähler metric if and only if the corresponding scalar curvature $R_{E}$ is an affine function. Note that in this case, $R_{E}$ is a priori determined by the Delzant polytope $P$, by the property that for any affine function $f$ on $P$, we have (see [9])

$$
\mathcal{L}(f)=2 \int f d \sigma-\int_{P} f R_{E} d \mu=0,
$$

where $d \mu$ is the standard Lebesgue measure on $P$ and $d \sigma$ is the induced boundary Lebesgue measure on $\partial P$ : on every facet $P_{i}$, we require that $d l_{i} \wedge d \sigma$ is $d \mu$ up to a sign.

In the case when $(X, L)$ is a compact polarized manifold, Yau [29], Tian [28] and Donaldson [9] conjectured

Conjecture 2.2. $(X, L)$ admits constant scalar curvature Kähler (cscK) metrics in $c_{1}(L)$ if and only if it is $K$-stable.

It is known by Donaldson [13], Stoppa [26], Mabuchi [23,24], Stoppa and Székelyhidi [27], Chen and Tian [8] that if $(X, L)$ admits cscK metrics in $c_{1}(L)$, then $(X, L)$ must be $K$-stable. In the product case $X=X_{1} \times X_{2}, L=L_{1} \otimes L_{2}$, one easily infers that each $\left(X_{i}, L_{i}\right)$ must be $K$-stable. Thus, Problem (1.1) would follow from Conjecture (2.2) by using the uniqueness of $\operatorname{cscK}$ metrics $[8,14]$. 
In [9], Donaldson considers the toric case and finds that the $K$-stability is related to the following condition:

Definition 2.3. A rational Delzant polytope is (relative) $K$-stable if for any convex continuous rational piecewise linear function $f$ one has $\mathcal{L}(f) \geq 0$. And the equality holds if and only if $f$ is an affine function.

He thus conjectures [9]:

Conjecture 2.4 (Donaldson). A compact toric Kähler manifold admits a compatible extremal metric if and only if $\mathcal{L}(f) \geq 0$ for any convex continuous piecewise linear function $f$ with equality if and only if $f$ is an affine function.

Once again, it is straightforward to see that if a product of two Delzant polytopes $P=P_{1} \times P_{2}$ is $K$-stable, such is then each factor $P_{i}$. However, as far as Conjecture (2.4) stays open, we must find an alternative argument to establish our Theorem (1.2). To this end, we will use the Calabi flow [4], which was initially introduces as a flow on the space $\mathcal{H}$ defined by

$$
\frac{\partial \varphi}{\partial t}=R_{\varphi}-\underline{R}
$$

where $R_{\varphi}$ is the scalar curvature of the Kähler metric $\omega_{\varphi}$ and $\underline{R}$ is a topological constant on $X$ defined by

$$
\underline{R}=\frac{2 n \pi c_{1}(X) \wedge[\omega]^{n-1}}{[\omega]^{n}} .
$$

In the toric case, this flow can be rewritten in terms of symplectic potentials as $([9])$

$$
\frac{\partial u}{\partial t}=\underline{R}-R_{u}
$$

We shall rather consider the modified version [21]

$$
\frac{\partial u}{\partial t}=R_{E}-R_{u}
$$

Note that by Chen and He [7], the Calabi flow exists for a short time starting from any $C^{3, \alpha}$ relative Kähler potential. Thus for a smooth symplectic potential $u$, the Calabi flow starting from $u$ also exists for a short time.

Guan [18] has shown that in the toric setting, for any two symplectic potentials $u_{1}$ and $u_{2}$, the geodesic in the sense of Mabuchi [22], Semmes [25] and Donaldson [15] connecting them is given by $(1-t) u_{1}+t u_{2}, t \in[0,1]$. The length of this geodesic is

$$
\sqrt{\int_{P}\left(u_{1}-u_{2}\right)^{2} d \mu}
$$

Suppose that $u_{1}(t), u_{2}(t), t \in[0,1]$ are two modified Calabi flows, we want to show that the geodesic distance between $u_{1}(t)$ and $u_{2}(t)$ decreases as $t$ increases. This is essentially known by the work of Calabi and Chen [6]. In fact, we have the following lemma:

\section{Lemma 2.5.}

$$
\frac{\partial}{\partial t} \int_{P}\left(u_{1}(t)-u_{2}(t)\right)^{2} d \mu \leq 0
$$


Proof.

$$
\begin{aligned}
\frac{\partial}{\partial t} \int_{P}\left(u_{1}(t)-u_{2}(t)\right)^{2} d \mu & =2 \int_{P}\left(u_{1}(t)-u_{2}(t)\right)\left(R_{u_{2}(t)}-R_{u_{1}(t)}\right) d \mu \\
& =2 \int_{P}\left(u_{1}(t)_{i j}-u_{2}(t)_{i j}\right)\left(u_{1}(t)^{i j}-u_{2}(t)^{i j}\right) d \mu \quad(*) .
\end{aligned}
$$

In the last step, we have used integration by parts as in Lemma 3.3.5 of [9]. For any $x \in P$, let $A=\left(D^{2} u_{1}(t)\right)(x), B=\left(D^{2} u_{2}(t)\right)(x)$, then

$$
\left(u_{1}(t)_{i j}-u_{2}(t)_{i j}\right)\left(u_{1}(t)^{i j}-u_{2}(t)^{i j}\right)(x)=\operatorname{Trace}\left((A-B)\left(A^{-1}-B^{-1}\right)\right) .
$$

Note that $A, B$ are positive-definite matrices, thus there exists an orthonormal matrix $O_{1}$ such that $O_{1} A O_{1}^{t}$ is a diagonal matrix $\operatorname{diag}\left(\lambda_{1}, \ldots, \lambda_{n}\right)$. Let $O_{2}=\operatorname{diag}\left(\sqrt{\lambda_{1}}, \ldots\right.$, $\left.\sqrt{\lambda_{n}}\right)$. Then $O_{2}^{-1} O_{1} A O_{1}^{t} O_{2}^{-1}$ is the identity matrix and $\tilde{B}=O_{2}^{-1} O_{1} B O_{1}^{t} O_{2}^{-1}$ is still a positive-definite matrix. Note that

$$
\operatorname{Trace}\left((A-B)\left(A^{-1}-B^{-1}\right)\right)=\operatorname{Trace}\left(\left(I_{n}-\tilde{B}\right)\left(I_{n}-\tilde{B}^{-1}\right)\right) .
$$

We can again choose an orthonormal matrix $O_{3}$ such that $O_{3} \tilde{B} O_{3}^{t}$ is a diagonal matrix $\operatorname{diag}\left(\bar{\lambda}_{1}, \ldots, \bar{\lambda}_{n}\right)$. Then

$$
\operatorname{Trace}\left((A-B)\left(A^{-1}-B^{-1}\right)\right)=\sum_{i=1}^{n}\left(1-\bar{\lambda}_{i}\right)\left(1-\bar{\lambda}_{i}^{-1}\right) \leq 0 .
$$

Thus, $(*) \leq 0$.

\section{Proof of Theorem (1.2)}

Let $\left(X_{i}, \omega_{i}\right)$ be toric symplectic manifolds with Delzant polytopes $P_{i}$. The product manifold $X=X_{1} \times X_{2}$ with symplectic form $\omega=\omega_{1}+\omega_{2}$ is symplectic toric with Delzant polytope $P=P_{1} \times P_{2}$. In the symplectic side, we have symplectic potentials $u_{i}$ satisfying Guillemin boundary conditions of $P_{i}$. We let $x$ be the variable of $P_{1}$ and $y$ be variable of $P_{2}$. Our assumption shows that there exists a symplectic potential $u$ on $P$ and

$$
u(x, y)=u_{1}(x)+u_{2}(y)+f(x, y), \quad f(x, y) \in C^{\infty}(\bar{P})
$$

such that the scalar curvature of $u(x, y)$ is an affine function. Our goal is to show that $f(x, y)$ is separable. Let

$$
f_{1}(x)=\frac{1}{\operatorname{vol}\left(P_{2}\right)} \int_{P_{2}} f(x, y) d y, \quad f_{2}(y)=\frac{1}{\operatorname{vol}\left(P_{1}\right)} \int_{P_{1}} f(x, y) d x .
$$

Then we have

Proposition 3.1. $v(x, y)=u_{1}(x)+u_{2}(y)+f_{1}(x)+f_{2}(y)$ is a symplectic potential of $P$ satisfying the Guillemin boundary conditions.

Proof. It is easy to see that $f_{1}(x)+f_{2}(y)$ is a smooth function on $\bar{P}$. Thus, we only need to show that $\left(D^{2} v\right)$ is a positive matrix in order to prove that $v$ is a symplectic potential. To show $\left(D^{2} v\right)>0$ is equivalent to show that $\left(D^{2}\left(u_{1}(x)+f_{1}(x)\right)\right)>0$ and $\left(D^{2}\left(u_{2}(y)+f_{2}(y)\right)\right)>0$. However, $\left(D^{2}\left(u_{1}(x)+f_{1}(x)\right)\right)>0$ and $\left(D^{2}\left(u_{2}(y)+f_{2}(y)\right)\right)>0$ just follow from the fact that $\left(D^{2} u\right)>0$.

Let $\mathcal{S}$ be the set of all symplectic potentials. We define a subset of $\mathcal{S}$. 


\section{Definition 3.2.}

$$
\begin{gathered}
\mathcal{M}=\left\{\underline{u}(x, y) \in \mathcal{S} \mid \underline{u}(x, y)=u_{1}(x)+u_{2}(y)+g_{1}(x)+g_{2}(y)\right. \text { s.t. } \\
g_{1}(x) \in C^{\infty}\left(\bar{P}_{1}\right), \int_{P_{1}} f_{1}(x) d x=\int_{P_{1}} g_{1}(x) d x \\
\left.g_{2}(y) \in C^{\infty}\left(\bar{P}_{2}\right), \int_{P_{2}} f_{2}(y) d y=\int_{P_{2}} g_{2}(y) d y\right\} .
\end{gathered}
$$

Then we have

Proposition 3.3. For any $\underline{u} \in \mathcal{M}$, we have

$$
\int_{P}(u(x, y)-v(x, y))^{2} d x d y \leq \int_{P}(u(x, y)-\underline{u}(x, y))^{2} d x d y .
$$

And the equality holds if and only if $v=\underline{u}$.

Proof. (3.1) is equivalent to show that

$$
\int_{P}\left(f(x, y)-f_{1}(x)-f_{2}(y)\right)^{2} d x d y \leq \int_{P}\left(f(x, y)-g_{1}(x)-g_{2}(y)\right)^{2} d x d y .
$$

Expressing it out, we have

$$
\begin{aligned}
\int_{P}- & 2 f(x, y)\left(f_{1}(x)+f_{2}(y)\right)+f_{1}^{2}(x)+f_{2}^{2}(y) d x d y \\
& \leq \int_{P}-2 f(x, y)\left(g_{1}(x)+g_{2}(y)\right)+g_{1}^{2}(x)+g_{2}^{2}(y) d x d y
\end{aligned}
$$

which is equivalent to

$$
0 \leq \int_{P}\left(f_{1}(x)-g_{1}(x)\right)^{2}+\left(f_{2}(y)-g_{2}(y)\right)^{2} d x d y .
$$

The equality holds if and only if $f_{1}(x)=g_{1}(x)$ and $f_{2}(y)=g_{2}(y)$.

Proof of Theorem (1.2). We use the Calabi flow to show that $v$ is an extremal symplectic potential. Let $u(t)$ be a sequence of symplectic potentials satisfying the modified Calabi flow equation on $P$ and $u(0)=v$. By Lemma 2.5, we have

$$
\frac{d}{d t} \int_{P}(u(t)-u)^{2} d x d y \leq 0
$$

Since $u(t) \in \mathcal{M}$, we obtain $u(t)=v$. This shows that $v$ is a separable extremal symplectic potential on $P$. By the uniqueness of the extremal symplectic potential modulo affine functions [18], it follows that $u$ is also separable. So, $f$ is a separable function.

\section{Acknowledgments}

The author would like to thank the referees for their suggestions for improving the presentation of the paper. He especially wants to thank Vestislav Apostolov for his encouragement and support in research, also his kind help in revising the article. Thanks also go to Paul Gauduchon and Gábor Székelyhidi for stimulating discussions. The author's research is financially supported by the Fondation mathématique Jacques Hadamard. 


\section{References}

[1] M. Abreu, Kähler geometry of toric varieties and extremal metrics, International J. Math. 9 (1998), 641-651.

[2] M. Abreu, Kähler metrics on toric orbifolds, J. Differ. Geom. 58(1) (2001), 151-187.

[3] V. Apostolov and H.N. Huang, A splitting theorem for extremal Kähler metrics, preprint accepted by J. Geom. Anal. DOI: 10.1007/s12220-013-9417-6.

[4] E. Calabi, Extremal Kähler metric, in Seminar of Differential Geometry (S.T. Yau, ed.) Annals of Mathematics Studies, 102, Princeton University Press, 1982, 259-290.

[5] E. Calabi, Extremal Kähler metric, II, in Differential Geometry and Complex Analysis (I. Chavel and H.M. Farkas, eds), Springer-Verlag, (1985), 95-114.

[6] E. Calabi and X.X. Chen, Space of Kähler metrics and Calabi flow, J. Differ. Geom. 61(2) (2002), 173-193.

[7] X.X. Chen and W.Y. He, On the Calabi flow, Amer. J. Math. 130(2) (2008), 539-570.

[8] X.X. Chen and G. Tian Geometry of Kähler metrics and holomorphic foliation by discs, Publ. Math. Inst. Hautes Étud. Sci. 107 (2008), 1-107.

[9] S.K. Donaldson, Scalar curvature and stability of toric varieties, J. Differ. Geom. 62 (2002), $289-349$.

[10] S.K. Donaldson, Interior estimates for solutions of Abreu's equation, Collectanea Math. 56 (2005), 103-142.

[11] S.K. Donaldson, Extremal metrics on toric surfaces: a continuity method, J. Differ. Geom. 79(3) (2008), 389-432.

[12] S.K. Donaldson, Constant scalar curvature metrics on toric surfaces, Geom. Funct. Anal. 19(1) (2009), 83-136.

[13] S.K. Donaldson, Lower bounds on the Calabi functional, J. Differ. Geom. 70 (2005), 453-472.

[14] S.K. Donaldson, Scalar curvature and projective embeddings, I, J. Differ. Geom. 59 (2001), $479-522$.

[15] S.K. Donaldson, Symmetric spaces, Kähler geometry, and Hamiltonian dynamics, Amer. Math. Soc. Transl. 196 (1999), 13-33.

[16] T. Delzant, Hamiltoniens périodiques et images convexes de l'application moment, Bull. Soc. Math. France, 116(3) (1988), 315-339.

[17] R.J. Feng and H.N. Huang, The global existence and convergence of the Calabi flow on $\mathbb{C}^{n} / \mathbb{Z}^{n}+$ $i \mathbb{Z}^{n}$, J. Funct. Anal. 263(4) (2012), 1129-1146.

[18] Z. Guan, On modified Mabuchi functional and Mabuchi moduli spaces of Kähler metrics on toric bundles, Math. Res. Lett. 6 (1999), 547-555.

[19] V. Guillemin, Kaehler structures on toric varieties, J. Differ. Geom. 40 (1994), 285-309.

[20] V. Guillemin, Moment maps and combinatorial invariants of Hamiltonian $T^{n}-$ spaces, Birkhauser, 1994.

[21] H.N. Huang and K. Zheng, Stability of Calabi flow near an extremal metric, Ann. Sci. Norm. Super. Pisa Cl. Sci. (5) 11(1) (2012), 167-175.

[22] T. Mabuchi, Some symplectic geometry on compact Kähler manifolds I, Osaka J. Math, (24) 1987, 227-252.

[23] T. Mabuchi, K-stability of constant scalar curvature polarization, preprint 2008, arXiv:0812.4093.

[24] T. Mabuchi, A stronger concept of K-stability, preprint 2009, arXiv:0910.4617.

[25] S. Semmes, Complex Monge-Ampère equations and symplectic manifolds, Amer. J. Math. 114 (1992), 495-550.

[26] J. Stoppa, K-stability of constant scalar curvature Kähler manifolds, Adv. Math. 221(4) (2009), 1397-1408.

[27] J. Stoppa and G. Székelyhidi, Relative K-stability of extremal metrics, J. Eur. Math. Soc. 13(4) (2011), 899-909.

[28] G. Tian, Kähler-Einstein metrics of positive scalar curvature, Inventiones Math. 130 (1997), $1-57$.

[29] S.T. Yau, Review of Kähler-Einstein metrics in algebraic geometry, Israel Mathematics Conf. Proc., Bar-Ilan University 9 (1996), 433-443.

CMls, École Polytechnique, 91128 Palaiseau, France

E-mail address: hnhuang@gmail.com 